\title{
The Study on the Application of Computer Technology in Coal Quality Detection
}

\author{
DING Yang ${ }^{1, \text { a }}$, JING jianzhong $^{2}$, ZHAN chuanjun $^{3}$ \\ 1,2,3 Guizhou Agency of Quality Supervision and Inspection of Coal Product, Liupanshui city \\ 553001, China \\ adingyang@126.com
}

Keywords: Computer Technology; Computer Control System; PLC; Coal Quality Detection

\begin{abstract}
Coal quality detection is used to measure moisture in coal, ash content, volatile and quality percentage content of solid carbon, is an important way to understand the characteristics of coal, is also a fundamental basis of evaluation of coal quality. Coal quality analyzer is an important instrument of detecting coal, to control the environment after coal burning pollution, reduce energy consumption, to ensure safety in production has an important role. How to implement the coal quality analyzer to run automatically, this to improve the efficiency of coal quality analysis, the accuracy of plays an important role. This paper discusses the computer control system in coal quality control detection system, the application of full automatic analyzer.
\end{abstract}

\section{Introduction}

Coal quality detection is used to measure moisture in coal, ash content, volatile and quality percentage content of solid carbon, is an important way to understand the characteristics of coal, is also a fundamental basis of evaluation of coal quality. According to the measured results of detection can be a preliminary judgment and types of the properties of coal, coal processing effect and industrial applications [1]. In recent years, China's coal detector on the structure, such as automatic control technology has achieved rapid development, has been widely used in various industrial fields. Research and development based on national standard of high performance and practical coal quality detection technology and instrument has important theoretical significance and of great economic value, has become a research hotspot in the field of coal industry in our country and related [2]. And the use of computer to the analysis of the coal industry, can improve the efficiency of the measurement, and can effectively avoid the error, industrial analysis accuracy was improved obviously.

Computer control system (CCS) refers to the application of computer and related network, and with the aid of some auxiliary equipment to realize the effective for controlled object control system [3]. Control system constantly collecting all kinds of information of the object being controlled, through certain procedures for processing, then output the results, so as to realize to control the purpose of the control object. Is mainly composed of hardware and software two parts, the control system of the software system is mainly refers to the application software system, such as: coal quality analysis software is used, the main characteristics of coal quality information acquisition, control, decision-making procedures, such as output data analysis task.

\section{Technology related to coal quality detection}

Computer control system is the application of computer in control and with the help of a few auxiliary components associated with the object, in order to obtain a certain control purpose, constitute the system. Computers usually index word here, there may be various size, such as from tiny to large general or specialized computers [4]. Accessories mainly refers to the input/output interface, test device and execute device, etc. Contact of the controlled object and the relationship between components, can be a way of cable, such as by cable connection of the analog signal or digital signal; It can also be a wireless mode, such as infrared, microwave, radio waves, light waves, 
etc. Coal quality detection are commonly used by computer control program to realize the goal of automatic control process of coal quality analysis.

Single-chip microcomputer control. Single-chip microcomputer control system first by a machine sent down to control the electrical part of the operation instructions, then under a machine on the system internal electrical driver module a command control signals, the pulse motor receives the signal after, in the form of content began operation, under the direction of a signal to change the original traditional sports as is consistent with the command signal linear motion, thus achieve the goal of lifting operations. Facilities lifting lever use sensors to induced changes of the lifting position, PC according to the next bit machine deliver output voltage value to detect the movement of data processing analysis, so as to realize the automatic analyzer for the purpose of intelligent control.

Embedded control. Is a microprocessor, embedded control system of the core components in coal quality analyzer, PLC control chip are common, the chip by dynamic and static storage programming, then the additional calculator to all sorts of the pulse signal of the sensor output to count, according to the analysis of pulse rate, the results of the analysis of the size of the coal ash content value.

\section{The embedded PLC technology in coal quality detection}

Computer software development plan PLC embedded PLC system structure shown in Figure 1, the system is mainly to complete the ladder programming, Ladder instruction encoding tables, and other functions, to achieve responsible for the delivery of embedded processor users and PLC the control program [5]. PLC as the control core embedded processors, compile and execute the PLC user program, the use of FPGA circuit expansion I/O, I/O points and flexible. PC connected via CAN bus interface card and CAN bus, PC embedded microprocessors and CAN bus communication, embedded processors and I/O expansion module communication, I/O and expansion PLC systems. Embedded PLC Systems will develop and run a separate operation, the development of computer users use the ladder program, applications and computer (PC) design platform development process, the soft PLC systems in development of embedded systems, the user program, through CAN bus communication and computer control system module and soft PLC distributed control network.

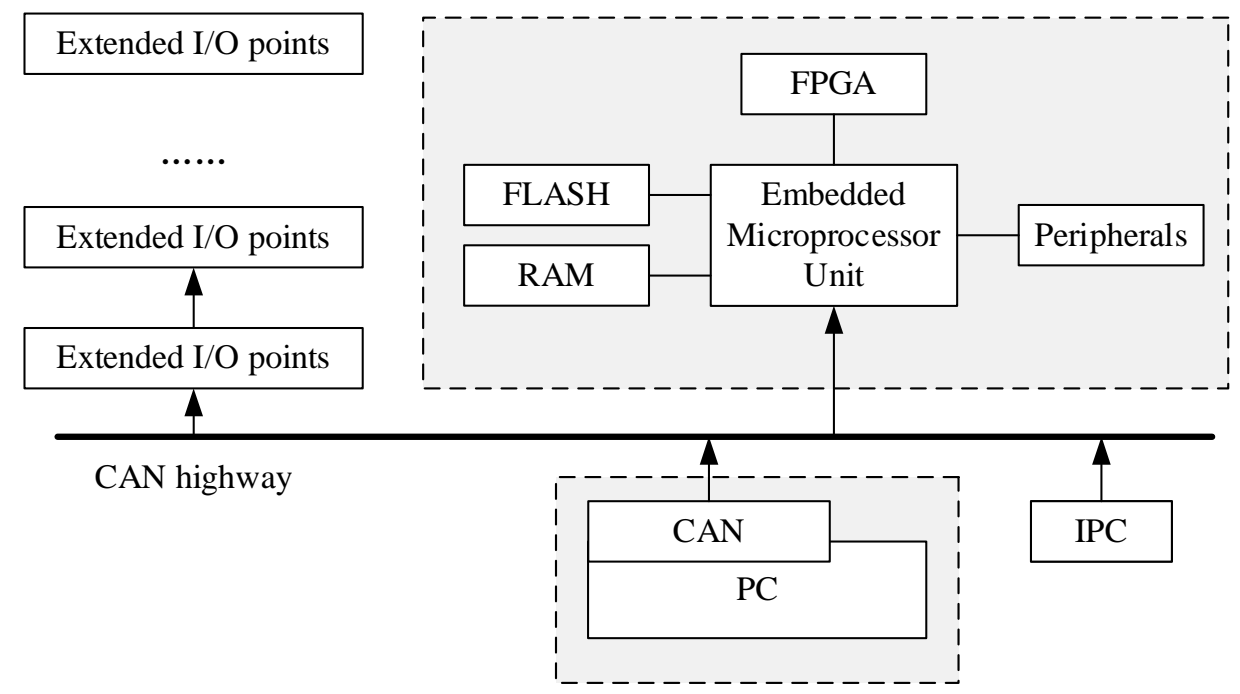

Figure 1. The embedded PLC system structure

Embedded PLC system can be divided into two parts: the development of systems and operational systems. Development system running on a PC platform, providing editing, compiling, debugging, simulation capabilities, and other features, the operating system running on embedded hardware platforms, responsible for analyzing the company's object code. Bridge object code is to develop systems and operational systems, the development of the system is connected to the PLC 
application written compiler to generate the appropriate profile connections, and then download the configuration file system operations, the operating system will parse the contents of the configuration file, use PLC to achieve a complete specific logic control.

\section{The development of coal quality detection control system}

The analysis of coal quality detection system. Coal quality detection analyzer is composed of multiple independent module, to achieve the effective connection between these modules, must use computer hardware and software control system can be achieved. Coal quality detection analyzer system hardware system is mainly composed of detection machine (including the furnace cover, furnace, rotary table, crucible, support bar and the thermocouple, etc.), a weighing module (high precision electronic scale, weighing lever, weighing plate as well as the crucible and so on), electrical module as well as the mechanical module (ball screw, polished rod, coupling, motor drives, PLC and touch screen, etc.), etc. Detection machine, weighing module, electrical module, and with the help of the computer network system control connection between mechanical module together, complete the whole process of coal, coal quality analysis. System software control system is mainly refers to the control program of PLC, touch screen, the function of the system software is mainly through the provision of man-machine control interface, realize the goal of control to the whole system, to ensure normal running of the automatic analyzer. System software system for acquisition to the coal ash content, volatile matter and related data for centralized treatment of water, and calculate the various components of concrete content. At the same time, through the computer auxiliary devices will detection the results of the output, so that the operator to save or print.

Coal quality analysis of the various modules of the control system of automatic operation is controlled by PLC, electric drive, gas and weighing control system by seamlessly between. Coal quality detection PLC control diagram is shown in figure 2.

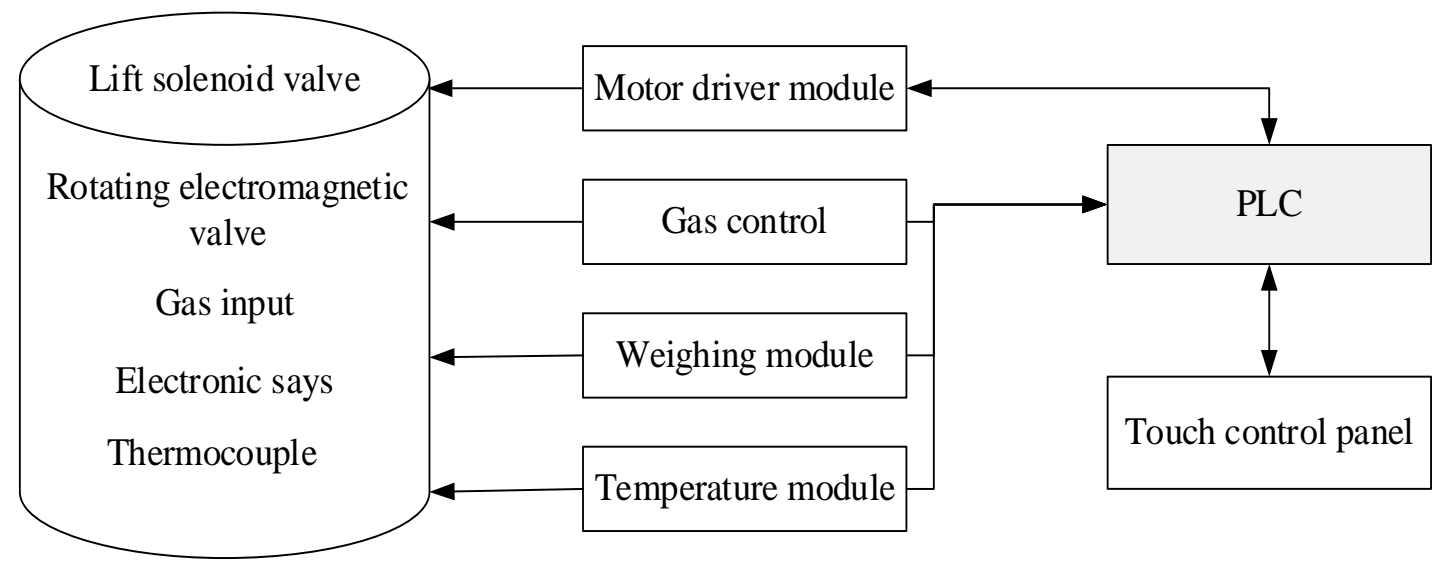

Figure 2. The coal quality detection control system

PLC as the core of the coal quality control detection system, the main operation, control, communications, programming, diagnosis and treatment functions. In the process of programming design, the connection between each node, logic must be strict, scientific, so as to ensure the safety of the system is running. To moisture, volatile matter, and ash in coal quality analysis detection, for example, automatic coal quality analyzer in the process of operation, not only should be rotating, lifting and so on each link (process) control, also for measurement, temperature control and the control of the gas proposed requirements accordingly. At the same time, according to the results of the automatic analyzer weighing scientifically calculated again, using the calculation results of difference to adjust the temperature of the input gas, to ensure that the coal quality detection control system under the computer control system safe, stable and efficient operation, the accuracy of measurement and ash content, moisture and volatile matter is higher also. 


\section{Conclusion}

Coal, coal quality analysis is to realize the industrial safety an important guarantee of safety production and residents. The traditional analysis method is mainly done by manual operation, deliver, sampling, and control, because the artificial operation in detection link prone to errors, such as coal quality analysis result accuracy is not high. In this paper, the application of computer in coal quality detection are discussed in this paper, through the use of computer control system for coal quality analyzer for automatic control, reduces the risk of artificial operation error occurred, coal quality analysis result is accurate and has higher reliability, in the coal ash content, moisture content and classification of volatile industry has important application value in the analysis.

\section{Reference}

[1] Yin W, Zhang L, Dong L: Applied spectroscopy, 2009, 63(8): 865-872.

[2] Othman M F, Shazali K: Procedia Engineering, 2012, 41: 1204-1210.

[3] YU Y, LIU Y, SUN X: Chinese Journal of Sensors and Actuators, 2010, 1: 005.

[4] Gao H, Liu S: Information and Computing Science, 2009. ICIC'09. Second International Conference on. IEEE, 2009, 2: 110-113.

[5] Zeng Q L, Wang J G, Wang L: Proceedings of the 2012 International Conference on Communication, Electronics and Automation Engineering. Springer Berlin Heidelberg, 2013: 187-191. 